\title{
Low-energy electron detection with delta-doped CCDs
}

\author{
Shouleh Nikzad ${ }^{a}$, Aimée L. Smith*, S.Tom Elliott ${ }^{a}$, Todd J. Jonesa ${ }^{a}$ T.A. Tombrellob, and Q. Yu ${ }^{a}$

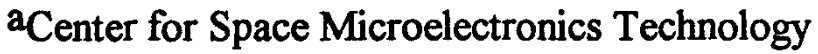 \\ Jet Propulsion Laboratory, California Institute of Technology, Pasadena, CA 91109 \\ bCalifornia Institute of Technology
}

\begin{abstract}
Delta-doped CCDs have achieved stable quantum efficiency, at the theoretical limit imposed by reflection from the Si surface in the near UV and visible. In this approach, an epitaxial silicon layer is grown on a fully-processed CCD using molecular beam epitaxy. During the silicon growth on the CCD, 30\% of a monolayer of boron atoms are deposited nominally within a single atomic layer, resulting in the effective elimination of the backside potential well.

In this paper, we will briefly discuss delta-doped CCDs and their application of to low-energy electron detection. We show that modification of the surface this way can greatly improve sensitivity to low-energy electrons. Measurements comparing the response of delta-doped CCDs with untreated CCDs were made in the $50 \mathrm{eV}-1.5 \mathrm{keV}$ energy range. For electrons with energies below $300 \mathrm{eV}$, the signal from untreated CCDs was below the detection limit for our apparatus, and data are presented only for the response of delta-doped CCDs at these energies. The effects of multiple electron hole pair (EHP) production and backscattering on the observed signals are discussed.
\end{abstract}

\section{LOW-ENERGY PARTICLE DETECTION AND CCDS}

Imaging systems for low-energy particles generally involve the use of microchannel plate electron multipliers followed by position sensitive solid state detectors, or phosphors and position sensitive photon detectors. These systems work well and can process up to 106 electrons/sec., however, the spatial resolution of these compound systems is considerably less than that of a directly imaged charge-coupled device (CCD). Also, these systems have difficulties with gain stability and they require high voltages. The present large format of CCDs, up to $4000 \times 4000$ pixels, could represent a major advance for the imaging of low energy particles. CCDs exhibit a highly linear response which is advantageous for quantitative detection applications. The full well capacity of buried channel CCDs corresponds to a collected electron density of about $10^{11}$ electrons $/ \mathrm{cm}^{2}$, which together with the low readout noise, gives CCDs a large dynamic range.

Frontside illumination of CCDs makes radiation of low penetration depth undetectable, because incident radiation is required to penetrate the CCD po;ycrystalline Si gates $(-5000 \AA)$,. One attempt to eliminate this problem involves turning the chip around in order to illuminate from the back side, thus eliminating attenuation due to the CCD processed layers. Backside illumination requires removal of the thick $\mathbf{p}^{+}$substrate in order to bring the exposed back surface in close proximity to the intended frontside potential well. However, thinning the CCD by chemically removing the substrate is not sufficient to obtain high quantum efficiency, because positive charge in the native oxide traps electrons generated near the back surface of the CCD. Termination of a Si surface with $\mathrm{SiO}_{2}$ leads to depletion of carriers at the surface, and in p-type Si the band bending due to surface depletion serves to create a surface potential well for electrons. This potential well can extend approximately $0.5 \mu \mathrm{m}$ into the p-doped epilayer which comprises the back surface of the thinned CCD, making the CCD insensitive to radiation which generates electrons near the surface. Moreover, the width of the potential well is sensitive to illumination, leading to hysteresis in the response of the thinned CCD. Electrons generated in this surface potential region, or diffusing to this region, recombine and are never detected. This problem is analogous to the detection of UV photons with silicon CCDs. Hoenk et al have successfully eliminated this effect for detection of UV light by MBE modifying the back surface with a ${ }^{++}$delta layer. Internal quantum efficiencies of unity were achieved ${ }^{1}$ in the UV as well as visible wavelength regimes, and stability over years has been demonstrated. 2 The $100 \%$ internal quantum efficiency implies the detection of every electron generated by UV photons that have penetration depths of $40-100 \AA$.

Low-energy electrons also have short penetration depths in Si and transfer a fraction of their energy to the crystal through electron-hole pair (EHP) production, motivating the attempt to extend application of the delta-doped CCD to direct electron imaging. Figure 1 shows the maximum penetration depth of electrons into silicon in the energy range $200 \mathrm{eV}$ to $5 \mathrm{keV}$ from Everhart et al. 3 As it is shown in the figure, the of the Previous work on electron detection with CCDs modified by ion implantation ${ }^{4}$ and flash gate treatment demonstrated sensitivity down to electron energies of $0.9 \mathrm{keV} .5$ Using deltadoped CCDs, we have successfully detected electrons down to $50 \mathrm{eV}$ with high efficiency. This paper will briefly discuss

* Present Address: Electronic Materials Group, MT, 77 Massachusetts Ave., Cambridge, MA, 02139 
the MBE modifications made to fully-processed CCDs and discuss the experimental results of application of the CCD to low energy electron detection.

Electrons with energies above $1.8 \mathrm{keV}$ are capable of generating $\mathrm{x}$-rays in silicon that can damage the gate oxide on the process-side of the device. While backside illumination provides some protection due to the 10-15 $\mu \mathrm{m}$ membrane of material between the region where incident electrons are likely to deposit their energy and the frontside gate oxide, low dark current for the device requires minimizing exposure to electrons of energy above $1.8 \mathrm{keV}$.

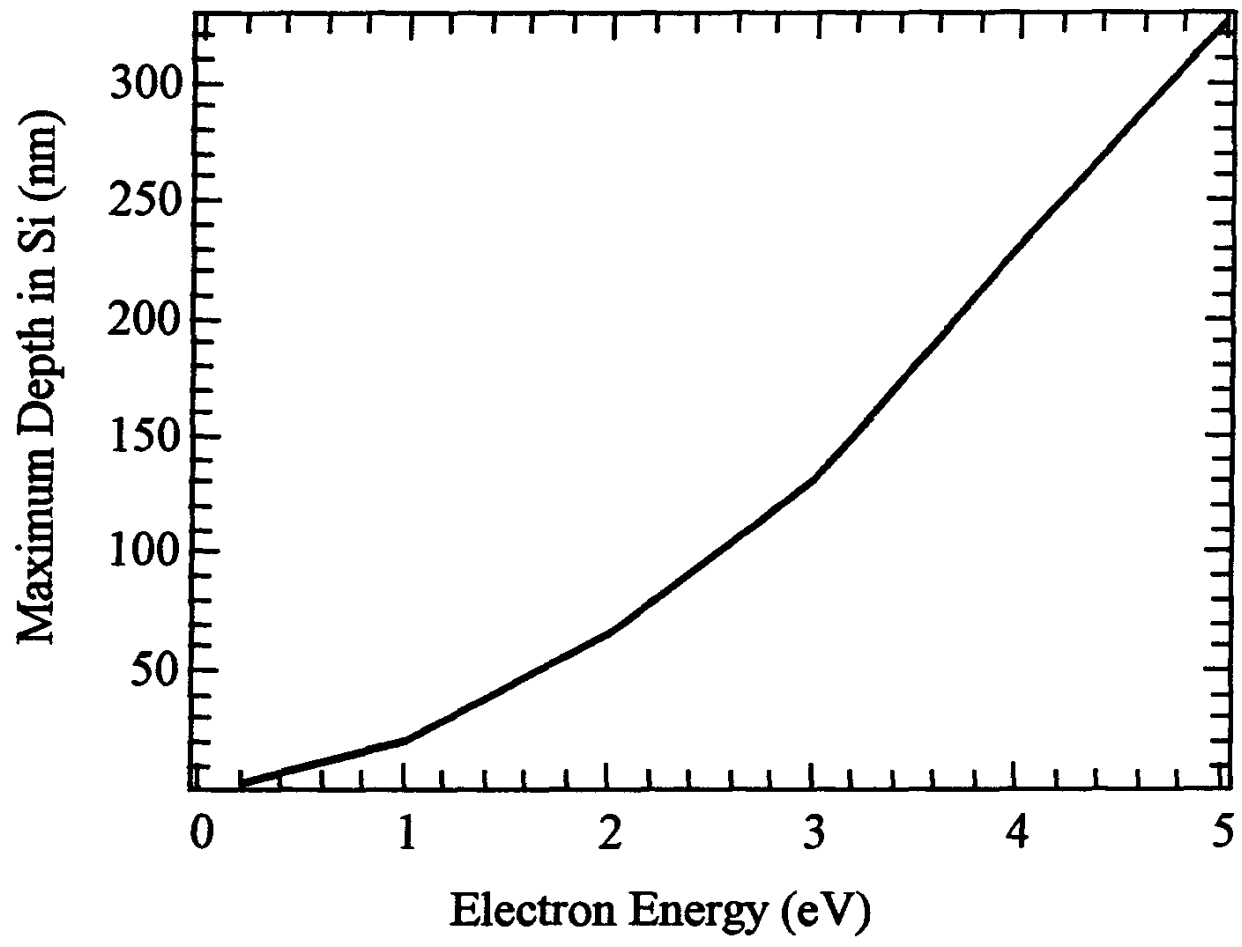

Fiqure 1. Maximum penetration depth in silicon for electrons in the energy range of $200 \mathrm{eV}$ to $5 \mathrm{keV}$ after Everhart et al. For energies below $1 \mathrm{keV}$, the electron penetration depth is less than $200 \AA$.

\section{DELTA-DOPED CCDS}

Delta-doped CCD processing is a recent development at JPL which uses MBE to enhance the UV response of backilluminated CCDs by removing the dead layer associated with these devices. The general processing procedure is as described by elsewhere. ${ }^{1,2}$ MBE modifications are made to the back surface of thinned, fully-processed CCDs by growing at low-temperature, $10 \AA$ of boron-doped Si followed by deposition of $2 \times 1014 \mathrm{~B} / \mathrm{cm}^{2}$, and a final $15 \AA$ layer of undoped silicon. MBE allows for the growth of atomically sharp, high concentration doping profiles and low-temperature growth ensures that the processing temperatures do not approach $500^{\circ} \mathrm{C}$, thereby avoiding dissolution of the silicon beneath the $\mathrm{Al}$ metallization, or spiking, of fully processed devices. During the in-situ preparation and subsequent MBE modification of the surface, the maximum temperature of the device is $450^{\circ} \mathrm{C}$ for a duration of four minutes. Boron diffusion is extremely slow at this temperature and therefore allows for an extremely thin layer of charge to be produced $5 \AA$ from the $\mathrm{Si} / \mathrm{SiO}_{2}$ interface. Figure 2 schematically shows the structure of delta-doped CCD back surface. TEM analysis has demonstrated that this low-temperature MBE modification is defect free and unlike ion implantation, will not require annealing to remove damage or to incorporate boron onto lattice sites. 6

Figure 3 shows a typical UV quantum efficiency of delta-doped CCDs. Note that the primary limitation to the internal quantum efficiency is the band structure near the back surface relative to absorption length of photons in silicon, so the most stringent test of the CCD quantum efficiency for photons is the $Q E$ at $270 \mathrm{~nm}$, where, the minimum absorption length occurs. Data taken at $270 \mathrm{~nm}$ shows that the device performs at $100 \%$ internal quantum efficiency at this worst-case wavelength, suggesting that at lower wavelengths and for the radiation with absorption length of this order, the response will be according to the predictions described in this section. Delta-doped CCDs have been extensively tested and have shown $100 \%$ internal quantum efficiency in the ultraviolet and visible part of the spectrum indicating that the deleterious backside potential well responsible for the detector dead layer has been effectively eliminated 


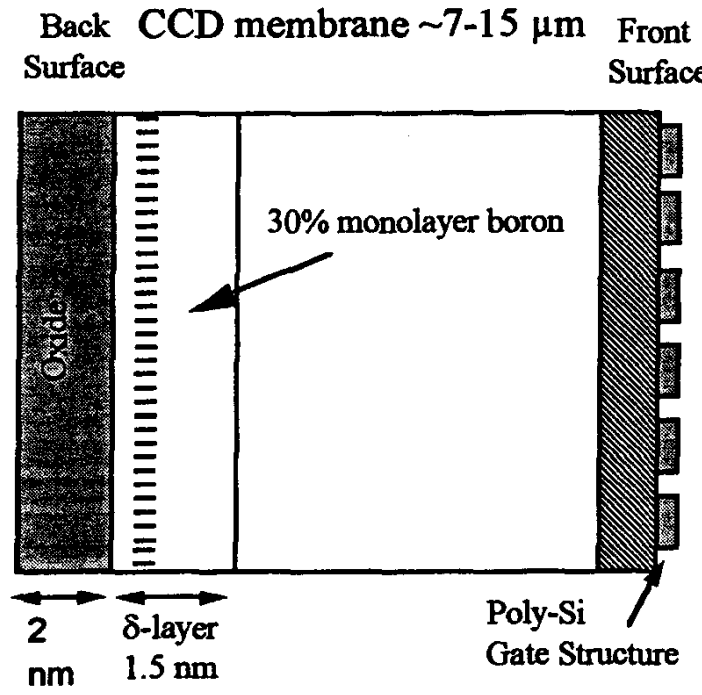

Figure 2 Schematic of the layer structure of a delta-doped CCD, shown in cross section. The layers added by MBE are a total of $2.5 \mathrm{~nm}$ in thickness, and contain $\sim 2.5 \times 10^{14}$ p-type dopant (boron) atoms.

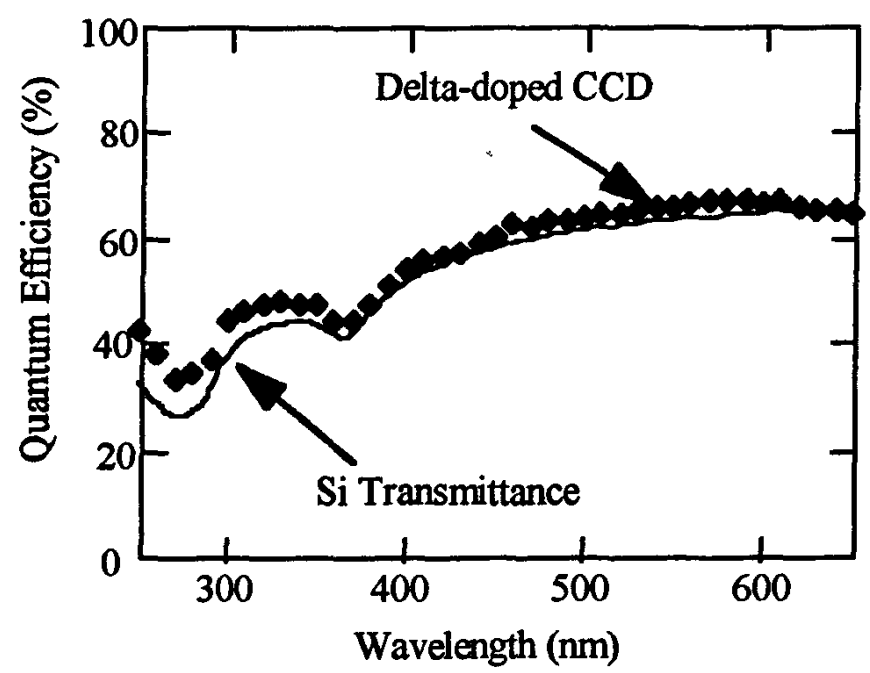

Fiqure 3 Typical quantum efficiency of delta-doped CCDs. Comparison with the reflection-limited quantum efficiency (transmittance of silicon ) shows that the delta-doped CCDs exhibit nearly $100 \%$ internal quantum efficiency.

\section{EXPERIMENTAL}

To gain an understanding of different aspects of low-energy electron response of delta-doped CCDs, we performed measurements using various electron sources and different device configurations. The various setups, electron sources, device configurations, and the specific points that can be gleaned from each measurement are described below. The CCDs used in these experiments were thinned, back-illuminated EG\&G Reticon CCDs. All measurements were repeated with both delta-doped and untreated CCDs. In some of the measurements, direct comparisons of delta-doped CCDs with untreated CCDs were made on the same device, using a delta-doped CCD which included a controlled (untreated) region. The controlled region was provided on the back surface of the array by masking off a portion of the surface during the MBE growth. All devices were fully-characterized prior to the electron measurements using UV illumination. Due to enhancement of quantum efficiency $(\mathrm{QE})$ in the $\mathrm{UV}$ by the delta-doping process, the untreated region of the partially deltadoped device were readily apparent as dark regions in the image made with uniform exposure to incident light radiation, i.e. flat-field exposure, using $250 \mathrm{~nm}$ photons. For $250 \mathrm{~nm}$ light, with absorption length of approximately $70 \AA$ in silicon, 7 the untreated region exhibited zero quantum efficiency whereas the delta-doped region exhibited reflection-limited response.

\section{SEM MEASUREMENTS}

One set of measurements was performed in an SEM to take advantage of its highly-focused electron beam. The SEM apparatus was a JEOL, model JSM 6400, and the measurements were made with beam energies ranging between $200 \mathrm{eV}$ and $1 \mathrm{keV}$. While it was not possible for modifications to be made to the SEM in order to accommodate the electronics necessary for collecting CCD images, performing photo-diode mode measurements was quite straightforward and informative. A CCD can be operated in such a way as to integrate the entire signal collected over the surface of the device, photo-diode mode, by grounding all pins except for the output amplifiers. The signal is then read from the pin of one of the output amplifiers, giving the compounded response of each of the pixels in the irradiated region of the device. Photo-diode mode measurements indicate the integrated response of the CCD to incident radiation and demonstrate the effect of the delta-doping treatment on overall collection efficiency. The fact that these measurements compound the response of all irradiated pixels into one measurement effectively averages out much of the error that would result in a pixel by pixel measurement. With the highly-focused beam of the SEM, we were able to make measurements in the untreated region as well as delta-doped regions and therefore directly observe the effect of the delta-doping process on collection efficiency. For each position measured on the surface of the device and for each energy, beam currents were first measured with a Faraday cup. CCD response to the electron beam at each position was measured in photo-diode mode, and finally, the beam current was again measured with the faraday cup to insure the stability of the beam current. Since the CCD is very sensitive to background light, response of the $\mathrm{CDD}$ was measured while deflecting the electron beam and it was found to be negligible. 


\section{UHV MEASUREMENTS}

A custom UHV system was used to make measurements in both photo-diode and the imaging mode. A schematic view of these two configurations is shown in figures $4 \mathrm{a}$ and $4 \mathrm{~b}$. For the photodiode mode measurements (shown in fig. 4a), each CCD in turn was mounted in plane with a Faraday cup and a phosphor screen onto a manipulator.

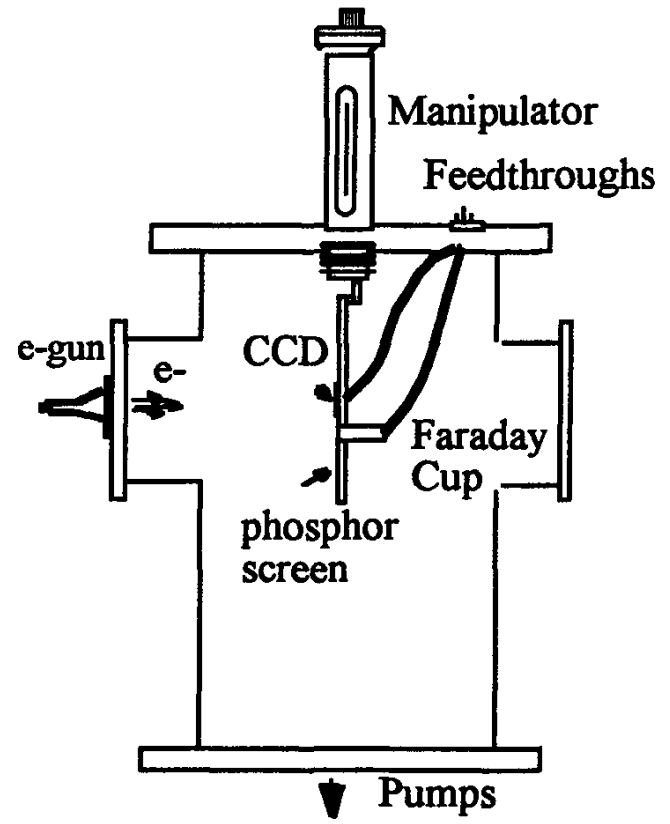

Figure 4a. UHV chamber used in the photodiode mode.

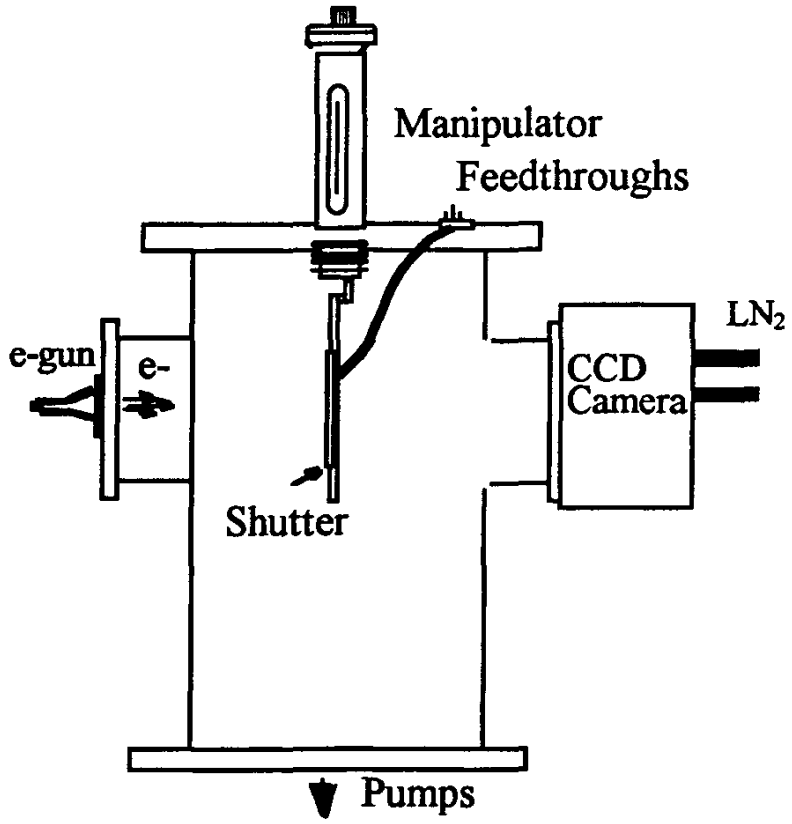

Figure $4 \mathrm{~b}$. UHV chamber used in the imaging mode.

Using the custom UHV system afforded the use of two different electron sources, one of very low energy and one of similar energies as used in the SEM measurements. The low-energy electron gun is a hot-filament cathode that produces electron energies of several $10 \mathrm{eV}$ while generating a strong light background. Comparison was made between the observed response of the CCD and the response of the CCD with the electron beam magnetically deflected. Because of the strong CCD response to the background light measurements with this electron gun beam are reported only qualitatively. The higher energy electron source which is a modified cathode ray tube (CRT) has reasonably stable beam energies varying from $300 \mathrm{eV}$ to several keV. Photo-diode mode measurements were made with beam energies ranging from $300 \mathrm{eV}$ to $1000 \mathrm{eV}$. Because it is an indirectly-heated cathode, this gun has very small background light, as was verified with our measurements. This background illumination was quantified by magnetically deflecting the electron beam. Repeated measurements were made on each CCD with calibration of the beam current in the Faraday cup both before and after each CCD measurement to insure beam stability. In this chamber geometry the beam spot was about one centimeter in diameter at the CCD. A circular aperture of $0.64 \mathrm{~cm}$ diameter (the same as the Faraday cup opening) was defined by a grounded aluminum sheet in front of the CCD to allow the exposure for the Faraday cup and the CCD to the same part of the electron beam.

In the imaging mode the electronics necessary for operating the CCD was attached to the chamber as shown in figure $4 \mathrm{~b}$. This mode of operation allows for observation of electron irradiation on operating parameters only apparent in imaging mode such as charge transfer efficiency (CTE), individual pixel response, and surface charging. For using the CCD in the imaging mode, we mounted a camera directly onto the UHV chamber. The electron source used for these measurements was the indirectly-heated cathode gun. Because of the highly-sensitive imaging mode of operation, the incoming flux of electrons was controlled by using a mechanical shutter thereby taking snap shots of the beam in $10 \mathrm{msec}$ to 2 second exposures. Preliminary measurements have been made at $500 \mathrm{eV}$ and more measurements are underway.

At electron beam energies lower than the silicon $\mathrm{K}_{\alpha}$ edge, there is no risk of damage to the silicon CCD due to the low absorption length of $\mathrm{x}$-rays in silicon for this energy range. Electrons at energies higher than approximately $1.8 \mathrm{keV}$ are capable of producing silicon $K_{\alpha}$ X-rays, which can penetrate the $\sim 10-15 \mu \mathrm{m}$ silicon membrane and damage the sensitive gate oxide on the front surface of the CCD. We verified the CCD's high tolerance to electrons at energies below the silicon 
$\mathrm{K}_{\alpha}$ edge by exposing the delta-doped $\mathrm{CCD}$ to $1.5 \mathrm{keV}$ electrons for several hours. Extensive UV testing was performed after this exposure as a test of effect of electron beam on the delta-doping treatment. No degradation of device performance was observed to result from exposure to electrons.

\section{RESULTS}

Figure 5 show the summary of the SEM and UHV measurements. Quantum efficiency was calculated by dividing the measured current from the CCD configured in photodiode mode to the measured electron beam current, which is equivalent to the number of electron-hole pairs generated divided by the number of incident electrons. Because portions of the deltadoped CCD were masked during processing to serve as control regions, data taken in the UHV system were corrected to account for the fraction of untreated exposed CCD area. Due to the negligible response of the untreated back-illuminated CCD at these energies, it was assumed that the control region of the delta-doped CCD does not contribute to the signal. The measured quantum efficiency of the delta-doped CCD increases with increasing energy of the incident beam. The dependence of quantum efficiency on incident energy is due to the complicated interaction of electrons with silicon which results in the generation of multiple electron-hole pairs in the cascade initiated by each incident electron. A significant fraction of the incident energy is undetected, due to backscattering of incident electrons and other energy dissipation mechanisms (e.g., secondary and Auger electron emission), as discussed in the next section. Multiple electron-hole pair production, also known in the literature as quantum yield, is also observed in the measured UV and x-ray response of deltadoped CCDs at photon energies greater than $\sim 3.5 \mathrm{eV}$. Quantum yield greater than unity has been previously observed in backside-illuminated CCDs modified using the flashgate ${ }^{3}$ and ion implantation ${ }^{8}$ at electron energies greater than $1 \mathrm{keV}$. Further discussion follow in the next section.

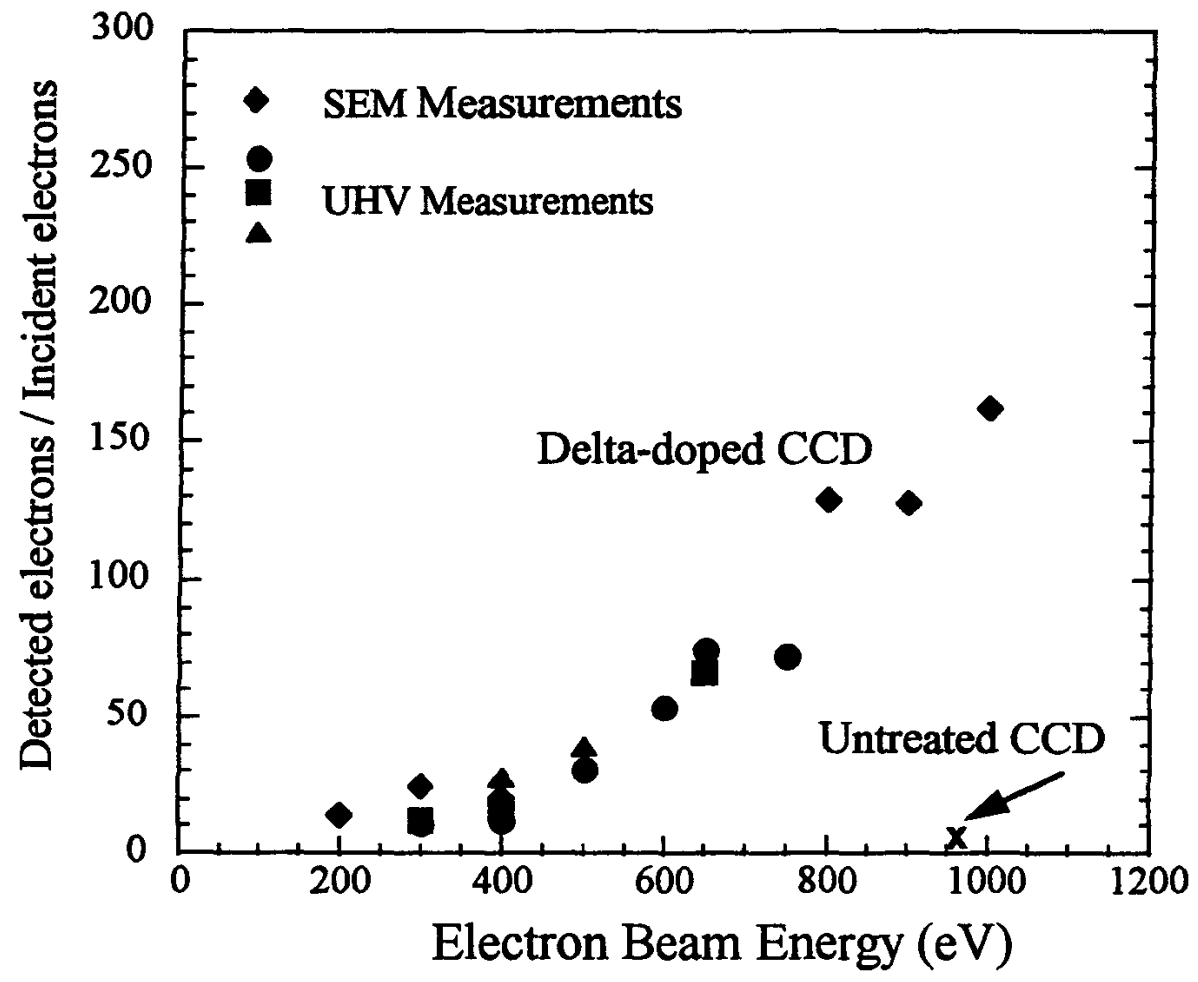

Figure 5. Quantum
efficiency of delta-
doped CCD with low-
energy electrons using
SEM and a indirectly-
heated cathode gun.
The increase in the QE
with energy is the
result of multiple
electron-hole pair
generation. The
response of the
untreated CCD has
been plotted for
comparison.

comparison.

The delta-doped CCD responds reliably and efficiently to electrons with energies lower than $0.9 \mathrm{keV}$. Moreover, at the previously reported lower limit of $900 \mathrm{eV}$ and $1 \mathrm{keV}$, the quantum efficiency of the delta-doped CCD is approximately twice that of the flashgate CCD. In the UHV chamber, the untreated backside-thinned CCD showed a dramatically lower quantum efficiency than the delta-doped CCD. The response of the untreated CCD to electrons was unstable, decaying with a time constant on the order of 20 minutes at an incident electron energy of $1 \mathrm{keV}$. This decay was not reversible by a thermal anneal at $90^{\circ} \mathrm{C}$ for several hours. In the SEM, the control regions of the delta-doped CCD showed no response to electrons at energies less than $300 \mathrm{eV}$. Even at $1 \mathrm{keV}$, the response was very low and unstable in these control regions. The delta-doped CCD exhibited a response above the noise at energies as low as $50 \mathrm{eV}$, using electrons from a directly heated filament source. In measurements with the hot filament, the electron signal was distinguished from the background light signal by measuring the CCD response before and after magnetically deflecting the electron beam. 
In preliminary measurements conducted in our laboratory, we report the first use of CCDs to image electrons. Flat-field images of $500 \mathrm{eV}$ electrons with the delta-doped CCD show excellent qualitative similarity to UV images at $250 \mathrm{~nm}$, with nearly identical contrast between delta-doped and control regions of the CCD. Some small dark blemishes are apparent in one corner of the electron flat-field image that are not seen on the UV flat-field, but this could be due to dust or debris that has been introduced to the membrane surface in the course of handling, transporting, and storing the device in the months following the date when the UV flat-field image was taken. Additional studies of electron imaging with the delta-doped CCD are under way.

\section{DISCUSSION}

In the ultraviolet, the measured quantum efficiency of a CCD is the product of three important quantities: the transmission coefficient, the quantum yield, and the internal quantum efficiency of the CCD. ${ }^{2}$ The transmission coefficient accounts for reflection from the surface and absorption in the native oxide, the quantum yield accounts for the statistically-averaged number of electron-hole pairs produced at the energy of the incident photon, and the internal quantum efficiency accounts for internal losses in the CCD, such as recombination of electron-hole pairs at the back surface of the CCD. Ultraviolet measurements of the delta-doped CCD indicate that the internal quantum efficiency is very nearly $100 \%$, even at $270 \mathrm{~nm}$ where the absorption length in silicon is only $4 \mathrm{~nm}$. The UV data suggest that the internal quantum efficiency of the deltadoped CCD is approximately $100 \%$ for electrons-provided the CCD is not damaged during the measurements. As discussed in the experimental section, we verified that the electron exposure did not degrade the performance of the CCD.

Incident electron radiation deposits energy in semiconductors through low-energy processes. Some of these mechanisms include secondary electron generation, Auger processes, Compton scattering, and backscattering. Part of the incident electron energy is transferred to the semiconductor through generation of EHPs. The average fraction of energy dissipated through these processes, EHP generation and all other losses, is a characteristic of the material. 9 For silicon, the statistical average number of EHPs generated by high-energy electrons or photons, also known as quantum yield, can be estimated by dividing the incident energy by $3.63 \mathrm{eV}$ over a wide range of incident energies. 10 The quantum yield has been measured for silicon using x-ray and ultraviolet radiation. The quantum yield for low-energy electrons has never been measured.

Among the important factors that influence the observed response to incident electron irradiation is backscattering of electrons. A large fraction of electrons are lost in backscattering as energetic electrons impinge upon the surface of the material. It is therefore necessary to have a good estimate of the backscattering coefficient in order to interpret the measured CCD quantum efficiency. Theoretical and experimental studies, alike, have concentrated on the backscattering coefficient of higher energy electrons (generally for energies greater than 5 or $10 \mathrm{keV}$ ). Drescher et al. have measured backscattering of 10-25 keV electrons from silicon and aluminum targets ${ }^{11}$ and Darlington et al. have measured backscattering from aluminum of electrons of energies down to $0.5 \mathrm{keV} .12$ These are shown in figure 6 along with theoretical estimates from Staub et al. ${ }^{13}$ The theory does not correlate well with the low-energy Al measurements. An estimate for the low energy backscattering coefficients of Si can be obtained by using a fit to Darlington's experimental Al data and then extrapolating the fit to $200 \mathrm{eV}$. While using this model gives some qualitative indication of the effect of backscattering on quantum efficiency of the delta-doped CCD for low-energy electron irradiation, the backscattering coefficient of low-energy electrons from silicon has not yet been measured. Using the measured backscattering coefficient of $\mathrm{Al}$ as an estimate for silicon, we have estimated that the backscattering coefficient for silicon is approximately $40-50 \%$ in the 200-1500 eV energy range. Even after taking backscattering into account, we are not detecting enough electrons to give us one electron for every $3.63 \mathrm{eV}$ of incident energy. This means that either the actual quantum yield is lower for electrons in this energy range, (or $3.63 \mathrm{eV}$ does not apply in this range) or other electron interactions contribute significantly to the transmission factor for low-energy electrons.

Analogous to the UV quantum efficiency discussed above, our electron response measurements represent the product of the effective quantum yield, the transmission factor (a factor representing the fraction of incident beam absorbed in the device which includes backscattering coefficient), and the quantum efficiency of the device. Assuming that all the generated electrons are detected by the delta-doped CCD (internal QE 100\%), our measurements will represent the product of the effective quantum yield of silicon and the transmission factor for low-energy electrons. If the transmission factor is dominated by the backscattering coefficient, i.e., $40-50 \%$ for $200-1500 \mathrm{eV}$ electrons, we have measured the effective quantum yield.

While separating the effects of transmission and quantum yield is interesting from a theoretical standpoint, the convolution of the two, as measured in these experiments, is the quantity of interest for solid-state electron detectors. It is significant that no other solid-state devices detect low-energy electrons as efficiently as the delta-doped CCD, due to the presence of a dead layer near their surfaces. In addition to its high efficiency, the delta-doped CCD also has the capability to image lowenergy particles, which may prove valuable in energy-selective particle detector applications. 


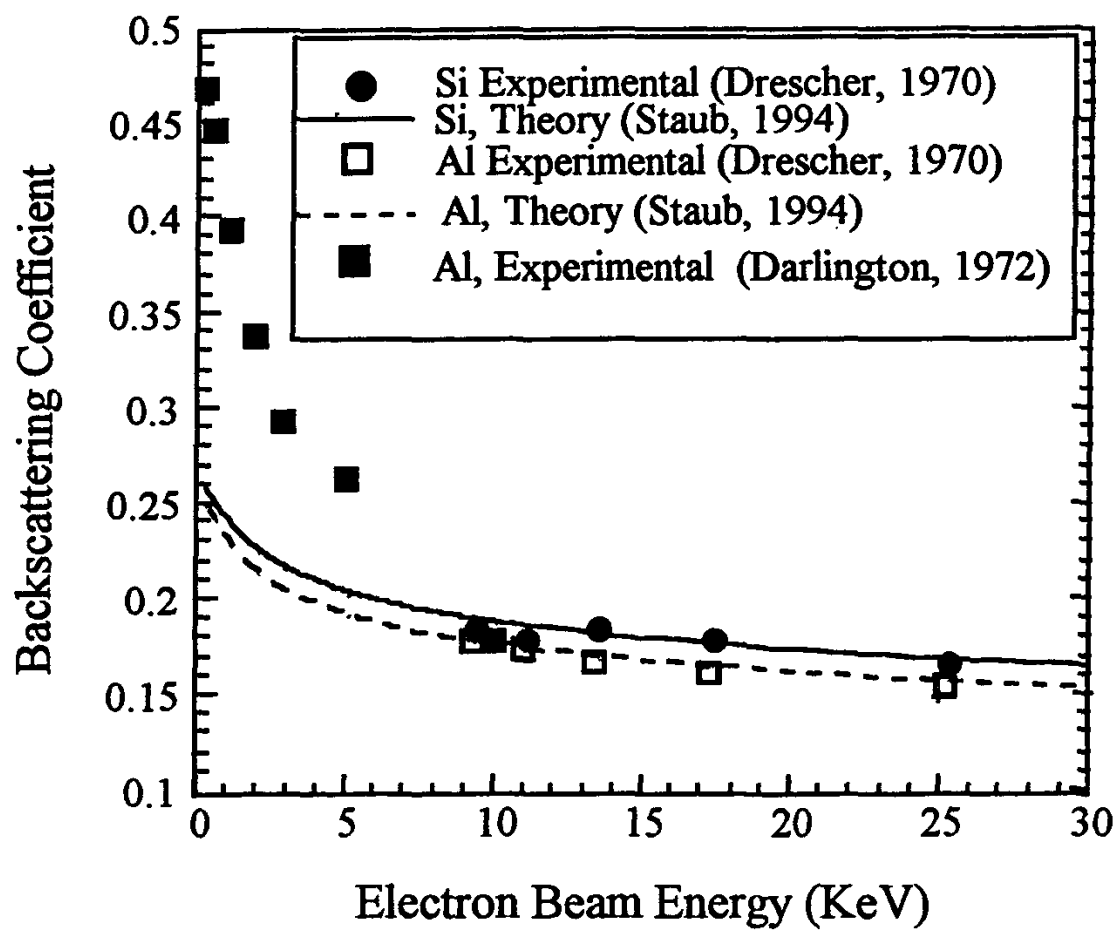

Fiqure 6. Experimental and theoretical backscattering results with extrapolation down to $200 \mathrm{eV}$.

\section{SUMMARY}

Because of their high resolution, linearity, and large dynamic range, CCDs could make major advances in particle detection. Delta-doped CCDs have been used for low-energy electron detection in the 50-1500 eV energy range, this represents the first measurements using CCDs to detect electrons in this energy range. Using delta-doped CCDs, we have extended the energy threshold for detection of electrons by approximately two orders of magnitude. We have also demonstrated the highest gain achieved to date by back-illuminated CCDs in response to low-energy electrons. Surface modification by delta-doping using MBE has demonstrated the highest quantum yield yet achieved for a backside electronirradiated CCD. For the first time, $500 \mathrm{eV}$ electrons were imaged with a delta-doped CCD.

\section{ACKNOWLEDGMENTS}

The authors gratefully acknowledge the invaluable assistance of Drs., L. Douglas Bell, Michael Hoenk, Steve Manion, Tom Van Zandt, Mr. Walter Proniawicz, and Professor L.C. Kimerling. The work presented in this paper was performed by the Center for Space Microelectronics Technology, Jet Propulsion Laboratory, California Institute of Technology, and was jointly funded by the Caltech President's fund and the NASA Office of Space Science.

\section{REFERENCES}

1. M.E. Hoenk, P.J. Grunthaner, F.J. Grunthaner, M. Fattahi, H.F. Tseng and R.W. Terhune, Appl. Phys. Lett., 61 (9) 1084 (1992).

2. S. Nikzad, M.E. Hoenk, P.J. Grunthaner, R.W. Terhune, R. Wizenread, M. Fattahi, H-.F. Tseng, and F.J. Grunthaner, Proc. of SPIE, 2217, Surveillance Technologies III, April 4-8, Orlando, Fl. (1994).

3. T.E. Everhart, and P.H. Hoff, J. Appl. Phys., 42 (13), 5837 (1971).

4. K.L. Luke and L.-J. Cheng, , J. Appl. Phys. 60, 589 (1986).

5. T. Daud, J.R. Janesick, K. Evans, and T. Elliott, Opt. Eng., 26 (8) 686 (1987). 
6. S. Nikzad, M.E. Hoenk, P.J. Grunthaner, R.W. Terhune and F.J. Grunthaner, Proc. SPIE, 2198, Astronomical Telescopes \& Instrumentation for the $21^{\text {st }}$ Century, March 13-18, Kona, Hawaii (1994).

7. S.M. Sze, Physics of Semiconductor Devices, $2^{\text {nd }}$ Ed, Wiley \& Sons, New York (1981) p.42

8. D.G. Stearns and J.D. Wiedwald, Rev. Sci. Instrum. 60 (6) 1095 (1989).

9. C.A. Klein, J. Appl. Phys. 39 (4) 2029 (1968).

10. L.R. Canfield, J. Kerner, and R. Korde, Appl. Opt. 283940 (1989).

11. H. Drescher, L. Reiner, and H. Seidel, Zeitschrift fur Angewandte Physik, 29 (6) 331(1970).

12. E.H. Darlington and V.E. Cosslett, J. Phys. D: Appl. Phys., 5 (11) 1969 (1972).

13. P. F. Staub, J. Phys D: Appl. Phys, 27 (7) 1533 (1994). 